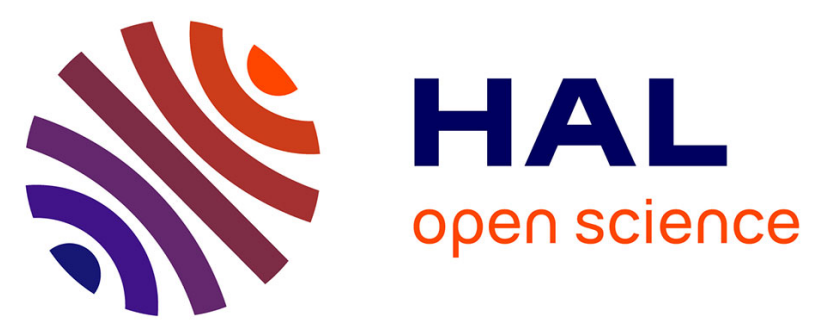

\title{
Spatially and temporally resolved optical spectroscopic investigations inside a self-pulsing micro thin-cathode discharge
}

Beilei Du, Mitsutoshi Aramaki, Sebastian Mohr, Yusuf Celik, Dirk Luggenhölscher, Uwe Czarnetzki

\section{To cite this version:}

Beilei Du, Mitsutoshi Aramaki, Sebastian Mohr, Yusuf Celik, Dirk Luggenhölscher, et al.. Spatially and temporally resolved optical spectroscopic investigations inside a self-pulsing micro thincathode discharge. Journal of Physics D: Applied Physics, 2011, 11 (25), pp.252001. 10.1088/0022$3727 / 44 / 25 / 252001$. hal-00627536

\section{HAL Id: hal-00627536 \\ https://hal.science/hal-00627536}

Submitted on 29 Sep 2011

HAL is a multi-disciplinary open access archive for the deposit and dissemination of scientific research documents, whether they are published or not. The documents may come from teaching and research institutions in France or abroad, or from public or private research centers.
L'archive ouverte pluridisciplinaire HAL, est destinée au dépôt et à la diffusion de documents scientifiques de niveau recherche, publiés ou non, émanant des établissements d'enseignement et de recherche français ou étrangers, des laboratoires publics ou privés. 


\title{
Spatially and temporally resolved optical spectroscopic investigations inside a self-pulsing micro thin-cathode discharge
}

\author{
Beilei Du ${ }^{1}$, Mitsutoshi Aramaki ${ }^{2}$, Sebastian Mohr ${ }^{1}$, Yusuf \\ Celik $^{1}$, Dirk Luggenhölscher ${ }^{1}$, and Uwe Czarnetzki ${ }^{1}$ \\ ${ }^{1}$ Institute for Plasma and Atomic Physics, Ruhr-University Bochum, 44780 Bochum, \\ Germany \\ ${ }^{2}$ Department of Electrical Engineering and Computer Science, Nagoya University, \\ Nagoya 464-8603, Japan \\ E-mail: Beilei.Du@ep5.rub.de
}

\begin{abstract}
A fiber probe is developed to investigate the temporal development and the spatial distribution of the population density of argon $1 \mathrm{~s}_{5}$ state and the emission in the afterglow in a self-pulsing micro thin-cathode discharge ( $\mu$ TCD) at atmospheric pressure. The fiber probe with a diameter $(125 \mu \mathrm{m})$ of about half the discharge diameter $(200 \mu \mathrm{m})$ is moved along the discharge axis. A tunable diode laser beam at $696.73 \mathrm{~nm}$ at resonance with the argon $1 \mathrm{~s}_{5} \rightarrow 2 \mathrm{p}_{2}$ transition is coupled into the far end of the fiber probe. The spatially differentiated absorbance yields the axial distribution of the $1 \mathrm{~s}_{5}$ state. In addition, the fiber is used to determine similarly the spatially resolved optical emission of various argon lines. Both the emission and the absorption profiles confirm that, in the afterglow, the plasma is strongly localized inside the hole of the discharge. Qualitatively, the measurements agree very well with the results of a zero-dimensional simulation. The long living afterglow is related to the high population density of the metastable atoms.
\end{abstract}


In recent years, micro-plasmas have become an emerging field of both plasma research and application. In research, due to the extreme conditions of the microdischarges, spatially resolved investigations are mostly restricted. In micro-cavity discharges, the radial distributions can be obtained by magnification of the discharge cross section emission image [1,2], but the axial distributions are often unknown. Here, a new technique is developed and applied to the investigation of the axial distributions in a micro thin-cathode discharge $(\mu \mathrm{TCD})$ operated in the self-pulsing mode. In this mode, a repeated ignition of a nanosecond spark discharge occurs [2] with a surprisingly long lifetime of the afterglow of several hundred nanoseconds. A kinetic model was developed to identify the mechanisms for the long living afterglow [3]. The model indicates that the long living afterglow is a result of the high population density of the excited states, especially the metastable 1s states, the formation of excimers and the high gas temperature which effectively results in a low recombination rate. Here, the axial distribution and the temporal development of the metastable atoms of the $1 \mathrm{~s}_{5}$ state in the afterglow are determined from tunable diode laser absorption spectroscopic (TDLAS) measurements using an optical fiber probe. The tunable diode laser beam at $696.73 \mathrm{~nm}$ in resonance with the argon $1 \mathrm{~s}_{5} \rightarrow 2 \mathrm{p}_{2}$ transition is coupled into the far end of the fiber probe. The spatially differentiated absorbance yields the axial density distribution of the $1 \mathrm{~s}_{5}$ state. The measurement result confirms the high population density of the metastable state indicated by the simulation. In addition, the fiber probe is used to determine the spatially resolved optical emission of various argon lines. Both the emission profiles and the distribution of the metastable atoms confirm a strong localization of the plasma in the afterglow around the dielectric isolator between the electrodes.

The $\mu$ TCD has a sandwich structure. A thin dielectric layer (here: $100 \mu \mathrm{m}$ ) separates two electrodes (Figure 1). One electrode, which is used as the anode, is much thicker (here: $1 \mathrm{~mm}$ ) than the cathode. The cathode has the same thickness as the dielectric. The discharge develops in a hole with a diameter of $200 \mu \mathrm{m}$ penetrating the sandwich structure. Molybdenum and alumina were used for the electrodes and the dielectric layer, respectively. To provide a pure argon environment, the $\mu$ TCD was placed in a chamber. The cathode side of the discharge faces a mirror, which reflects the emission light of the discharge under 90 degrees to an achromatic lens fixed on the side wall of the chamber. The achromatic lens images the emission light onto an optical fiber, which can be coupled with an optical detector, e.g. a photomultiplier or a spectrograph, etc. The fiber probe was moved from the anode side into the chamber through a small hole with a diameter of $1.5 \mathrm{~mm}$. The fiber probe is made of a multimode optical fiber. The bare fiber (including the core, the cladding and the coating) has a diameter of $250 \mu \mathrm{m}$. On one end of the fiber the coating is removed for about $10 \mathrm{~mm}$. The cladding with the core with a diameter of $125 \mu \mathrm{m}$ is moved into the discharge along discharge axis. The bare fiber is fixed in a ceramic tube, which has a length of $20 \mathrm{~cm}$, an inner diameter of $300 \mu \mathrm{m}$, and an outer diameter of $1 \mathrm{~mm}$. The rest of the bare fiber is protected by a jacket tube. A holder fixes the fiber probe with a motorized translation 
stage with a step resolution of $0.1 \mu \mathrm{m}$. The remaining gap between the ceramic tube and the hole has a cross section of about $1 \mathrm{~mm}^{2}$. Due to the small gap, the air in the chamber was not evacuated by a vacuum pump but pushed through the gap out of the chamber by a argon flux of $900 \mathrm{sccm}$. The $\mu$ TCD was operated at atmospheric pressure. The gas purity was checked via the emission spectrum of the discharge.

The $\mu \mathrm{TCD}$ was charged via a $500 \mathrm{k} \Omega$ series resistor. A $100 \Omega$ resistor was connected in series with the anode to monitor the current. In the self-pulsing mode, the discharge current pulse cannot be directly measured. More details about the current measurement are described in [2]. A $700 \mathrm{~V}$ dc voltage with negative polarity was applied. The discharge operates in the spark self-pulsing mode with a self-pulsing frequency of about $10 \mathrm{kHz}$. To avoid an overheating of the electrodes, the applied voltage was pulsed with $10 \mathrm{~Hz}$ and with $0.3 \%$ duty cycle.

(a)

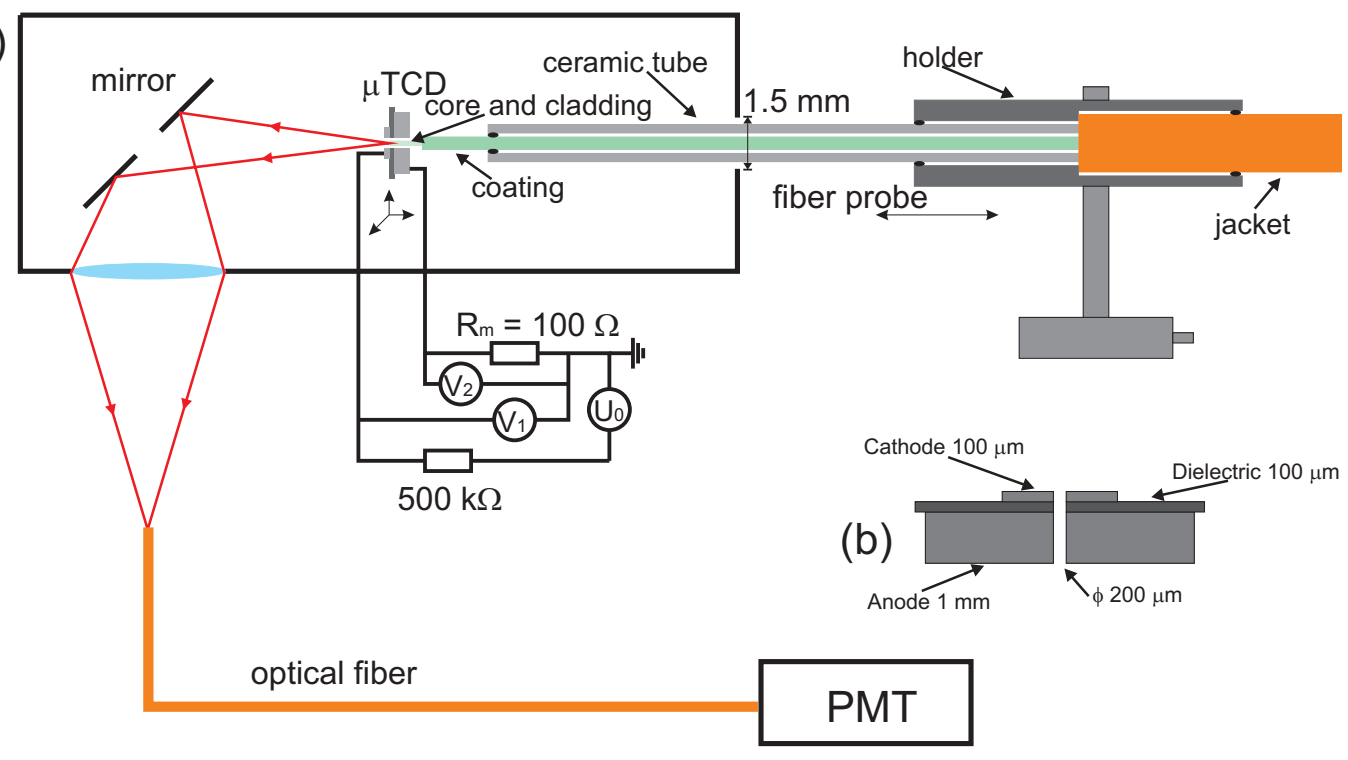

Figure 1. Experimental setup.

The density of the argon metastable atoms in the $1 \mathrm{~s}_{5}$ state was deduced from the TDLAS measurement. The laser system is described in detail in a recent work [4]. To avoid saturation of the absorption, the laser power is reduced to about $1.5 \mu \mathrm{W}$ by a neutral density filter. The laser beam is coupled into the far end of the fiber probe, then passes through the discharge, and is imaged onto the optical fiber connecting to a photomultiplier (Hamamatsu R928). An interference filter (FWHM $=10 \mathrm{~nm}$ ) is installed in front of the detector to discriminate the investigated spectral line. The signal of the photomultiplier is read out by an oscilloscope (LeCroy WaveSurfer 424), triggered by the discharge voltage pulse. The maximal output current of the photomultiplier is only $0.1 \mathrm{~mA}$. To intensify the voltage signal at the oscilloscope, while at the same time providing the temporal resolution, a $1 k \Omega$ terminal resistor was chosen, which lead to a response time of about $\tau=200 \mathrm{~ns}$. The real signal $S_{\text {real }}$ is recovered from the output 
signal $S$ by deconvolution:

$$
S_{r e a l}=S+\tau \dot{S}
$$

While moving the fiber probe along the discharge axis, the intensities of the laser radiation $I_{0, \nu}$, the plasma emission $I_{p l, \nu}$, and the laser radiation through the plasma emission $I_{l+p l, \nu}$ are recorded at each position and laser frequency with averaging over 200 plasma pulses. The intensity of the transmission $I_{\nu}$ is deduced by the difference of $I_{l+p l, \nu}$ and $I_{p l, \nu}$ :

$$
I_{\nu}=I_{l+p l, \nu}-I_{p l, \nu}
$$

and the absorbance $\int_{-\infty}^{+\infty} \ln \left(\frac{I_{0, \nu}}{I_{\nu}}\right) d \nu$ with respect of the probe position is obtained.

The difference in $I_{\nu}$ depends on the absorption coefficient $\alpha_{\nu}$ and the absorption length $d x$ :

$$
d I_{\nu}=-\alpha_{\nu} I_{\nu} d x \Longrightarrow \alpha_{\nu}=-\frac{d I_{\nu}}{I_{\nu} d x}=\frac{d}{d x} \ln \left(\frac{I_{0, \nu}}{I_{\nu}}\right)
$$

The absorption coefficient $\alpha_{\nu}$ depends on the density of the metastable atoms $n_{A r^{*}}[5]$ :

$$
\alpha_{\nu}=\frac{1}{4 \pi \varepsilon_{0}} \frac{\pi e^{2}}{m_{e} c} f n_{A r^{*}} g_{\nu}
$$

where $f=0.0313$ is the oscillator strength of the $1 \mathrm{~s}_{5} \rightarrow 2 \mathrm{p}_{2}$ transition [6] and $g_{\nu}$ is the normalized line profile. Equation (3) and Equation (4) lead to:

$$
\frac{d}{d x} \ln \left(\frac{I_{0, \nu}}{I_{\nu}}\right)=\frac{1}{4 \pi \varepsilon_{0}} \frac{\pi e^{2}}{m_{e} c} f n_{A r^{*}} g_{\nu}
$$

Integrating Equation (5) over the frequency, the spatially resolved metastable atom density is obtained by a simple differentiation of the measured absorbance:

$$
n_{A r^{*}}=4 \pi \varepsilon_{0} \frac{m_{e} c}{\pi e^{2}} \frac{1}{f} \frac{d}{d x} \int_{-\infty}^{+\infty} \ln \left(\frac{I_{0, \nu}}{I_{\nu}}\right) d \nu .
$$

Instead of coupling the laser into the fiber probe, the far end can also be connected to a $2 \mathrm{~m}$-spectrograph equipped with an intensified diode array with a gate-width of $33 \mathrm{~ms}$, much longer than the duration of the discharge. In this way, the fiber probe was used to perform time integrated and spatially resolved optical emission spectroscopic (OES) measurement, similar to an optical probe for spatially resolved OES described in [7]. Here, at the probe position $x$, the measured intensity $S(x)$ is given as an integral over the local emissivity $I(x)$ of the plasma:

$$
S(x)=\int_{x}^{+\infty} I\left(x^{\prime}\right) d x^{\prime}
$$

By a simple differentiation of the measured profile with respect to the probe position the local emissivity is deduced:

$$
I(x)=-\frac{d S(x)}{d x} .
$$




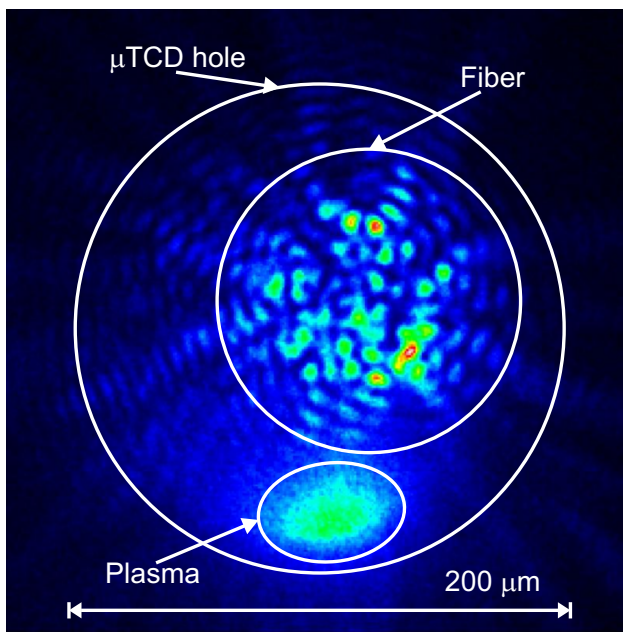

(a) The fiber probe is far from the cathode.

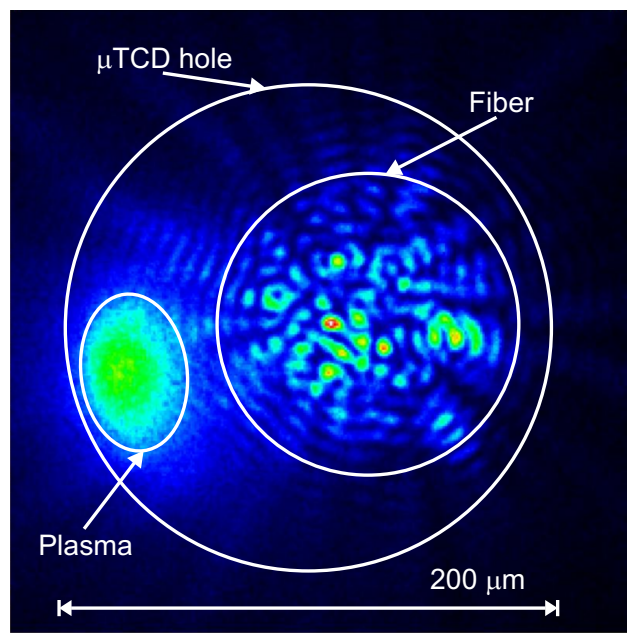

(b) The fiber probe is on the cathode surface.

Figure 2. ICCD images of the plasma emission and the laser beam from the fiber probe. White circles indicate the orifice of the discharge hole, the plasma volume and the beam out of the fiber probe.

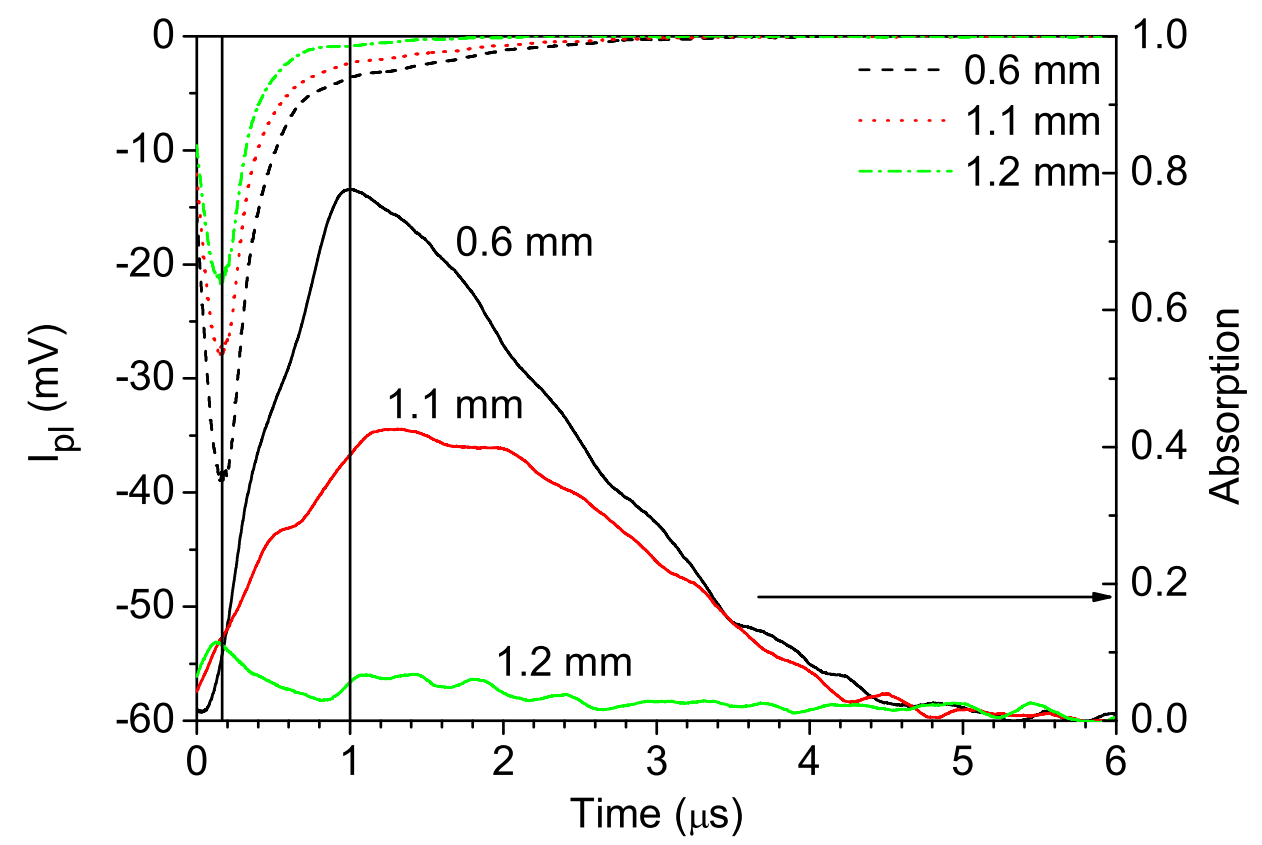

Figure 3. Measured plasma emission $I_{p l}$ (solid line) and the measured absorption (dashed line) with different fiber probe position along the discharge axis. The fiber probe position corresponds to the distance to the anode outer edge. $1.20 \mathrm{~mm}$ is at the cathode outer edge. 
In order to investigate a possible perturbance of the discharge by the presence of the fiber probe, various additional measurements were performed. Firstly, $V-I$ curves were recorded which show no influence by the presence of the fiber probe at all (breakdown voltage: $600 \mathrm{~V}$, current peak FWHM: $2 \mathrm{~ns}$, current amplitude: $36 \mathrm{~A}$ ). Secondly, the optical wave guide collecting the emitted radiation is replaced by an ICCD camera equipped with a microscope lens. Spatially resolved images are taken from the top of the discharge (Figure 2) with the integration time of $300 \mu$ s covering the first two selfpulsing sparks in a burst series. Clearly, the discharge is located as a tiny spot in the vicinity of the wall independent of the fiber probe position. The fiber image is blurred due to the speckle interference of the coherent diode laser beam. The strong localization of the discharge allows moving of the fiber probe along the axis without a significant disturbance of the plasma. On the other hand, in the absorption measurement, the main absorption may not be caused by the metastables within the localized plasma located at the wall. In fact, up to $80 \%$ absorption is observed which is only possible by the metastables covering the majority of the hole cross section. We will discuss this aspect in more detail in the later part of this paper.

The spectral absorption profiles were recorded at each position and time. They exhibit a Lorentz profile with a substantial broadening of about $11 \mathrm{pm}$. The FWHM of the pressure broadening is given by [9]:

$$
w_{\text {pressure }}=2 \gamma N \text {, }
$$

where $\mathrm{N}$ is the neutral gas density and $\gamma$ is the collision broadening coefficient given in [10]. For the $1 \mathrm{~s}_{5} \rightarrow 2 \mathrm{p}_{2}$ transition, $2 \gamma=0.910 \cdot 10^{-21} \mathrm{~nm} /$ atom $\mathrm{cm}^{3}$. Assuming the line broadening is only contributed by the pressure broadening, the neutral gas density of $\mathrm{Ar}$ is calculated to be $1.2 \cdot 10^{19} \mathrm{~cm}^{-3}$ relating to a gas temperature of about $600 \mathrm{~K}$ at atmospheric pressure. In the small spark created by the nanosecond current pulse, a higher gas temperature is expected. This indicates that the assumption of a gas temperature around $1000 \mathrm{~K}$ in the zero-dimensional simulation is reasonable.

To determine the absorption profiles, the cross section integrated plasma emission is recorded in parallel by the PMT as described above. The temporal development of the emission and absorption behavior at various fiber probe position is displayed in Figure 3. Apparently, emission decreases when the fiber is moved beyond the inner edge of the anode, which indicates shadowing. Further, the temporal development of the absorption profile changes. The absorption data are processed according to the procedure described above, and the result is summarized in Figure 4. The temporal development of the metastable density coincides with the temporal decay of the optical emission. An interpretation might be that the small spark created by the nanosecond current pulse at the edge is emitting high intensity VUV radiation which then leads to formation of the excited states over the full cross section of the discharge hole. Propagation of this VUV radiation is probably slowed down by radiation diffusion due to the very high optical density for ground state absorption at atmospheric pressure. These excited states relax to the metastable states which then causes the large volume 
diode laser beam absorption. In this model, the fiber will shadow the VUV radiation when moved beyond the cathode edge. i.e. causing a certain perturbation of the measured upper metastable densities. Nevertheless the measured $1 \mathrm{~s}_{5}$ state density of $6.3 \cdot 10^{14} \mathrm{~cm}^{-3}$ coincides well with he value calculated by the zero-dimensional model [3]. However, one has to be careful in the interpretation, since most likely the source of metastable production in the measurement is radiation and not the plasma directly.

In addition, two of the $2 \mathrm{p}_{2} \rightarrow 1_{\mathrm{s}_{5}}$ emission curves are displayed in Figure 4 . The lower left curve (the dashed line) shows the emission from the entire discharge without spatial resolution, recorded in parallel with the absorption. Connecting the fiber probe with the $2 \mathrm{~m}$-spectrograph, the axially resolved but time integrated emission is obtained. This curve is shown on the right part in Figure 4 (the dotted line). Clearly, metastables are localized mainly close to the inner edge of the cathode with a small peak close to the anode. The metastables appear with a substantial delay of about $0.5 \mu$ s and then last for several microseconds. The emission is localized between the anode and the cathode over a region of about $300 \mu \mathrm{m}$. The emission spectrum exhibits lines only after a certain temporal interval of about $200 \mathrm{~ns}$ after the nanosecond current pulse. At early times, the emission is dominated by a strong continuum which might be caused by bremsstrahlung. This continuum is also visible in the time integrated emission shown in Figure 5.

The power density in the discharge can be estimated from the measured volume of the emission (cross section diameter: $50 \mu \mathrm{m}$; length: $200 \mu \mathrm{m}$ ) and the stored energy of the capacitor $(120 \mathrm{pF})$ at the breakdown voltage of $600 \mathrm{~V}$. The corresponding power density in the $2 \mathrm{~ns}$ current pulse is $3 \cdot 10^{10} \mathrm{~W} / \mathrm{cm}^{3}$. These extreme values most likely cause a shock wave transversing the discharge in a similar time as the build up of the metastables. However, this shock wave can not be expected to transport metastables from the small spark through the highly collisional argon gas. However, it might be possible that the increased collisionality in the shock wave front might lead to quenching of the excited states and thereby causes metastable population.

In conclusion, in this work a simple optical fiber probe was applied to perform spatially and temporally resolved optical absorption and emission spectroscopic measurements along the discharge axis in the afterglow of a self-pulsing argon $\mu$ TCD. Both the emission profiles and the distribution of the metastable atoms indicate that in the afterglow the plasma is strongly localized around the dielectric isolator. Qualitatively, the measurement results agree very well with the zero-dimensional simulation results [3], where the long living afterglow is related to the high population density of the metastable atoms.

\section{Acknowledgments}

This work was supported by the "German Research Foundation" (DFG) in the frame of the "Research Unit FOR 1123 - Physics of Microplasmas", the Research Department "Plasmas with Complex Interaction" of the Ruhr-University Bochum, the Research School of the Ruhr-University Bochum, and the JSPS ITP program. The authors would 


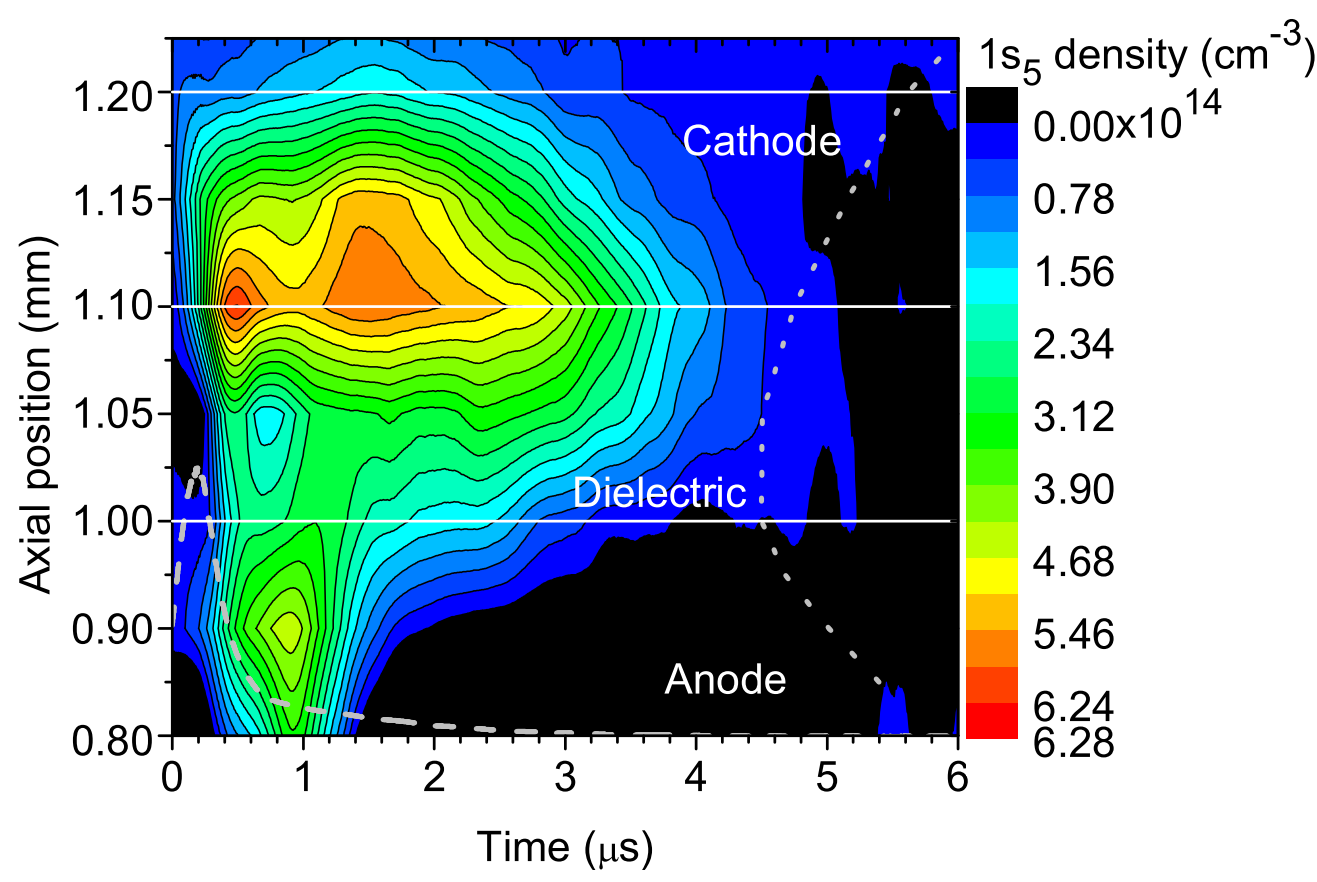

Figure 4. Color map: spatially (y-axis) and temporally (x-axis) resolved $1 \mathrm{~s}_{5}$ density. - - - : Temporal development of the $2 \mathrm{p}_{2} \rightarrow 1 \mathrm{~s}_{5}$ emission $(696.54 \mathrm{~nm})$ measured from the cathode side.

$\cdots \cdots$ : Time integrated spatial distribution of the $2 \mathrm{p}_{2} \rightarrow 1 \mathrm{~s}_{5}$ emission measured by the fiber probe.
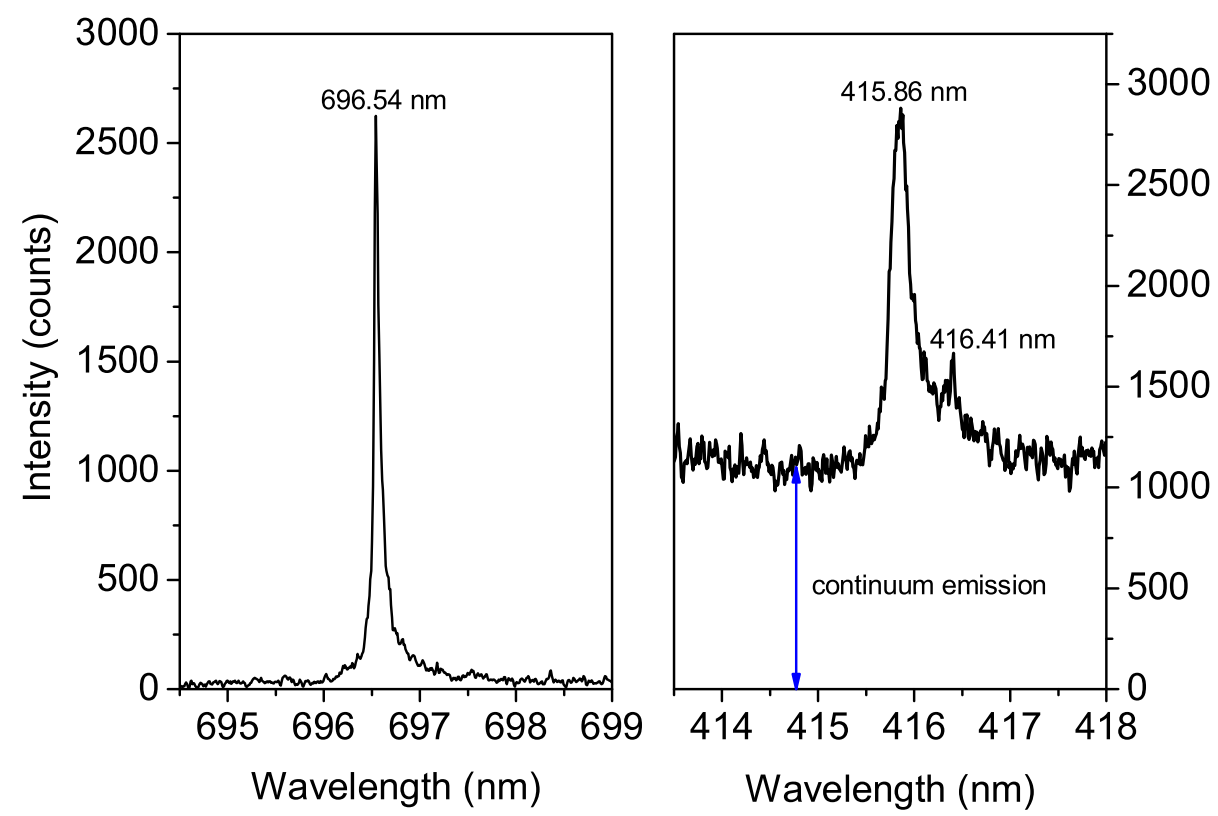

Figure 5. Time integrated emission spectrum measured with the fiber probe. The background has been subtracted. 
also like to thank Professor Igor Adamovich for the valuable suggestions.

\section{References}

[1] Lazzaroni C, Chabert P, Rousseau A and Sadeghi N 2010 J. Phys. D: Appl. Phys. 43124008

[2] Du B, Mohr S, Luggenhölscher D and Czarnetzki U 2011 J. Phys. D: Appl. Phys. 44125204

[3] Mohr S, Du B, Luggenhölscher D and Czarnetzki U 2010 J. Phys. D: Appl. Phys. 43295201

[4] Celik Y, Aramaki M, Luggenhölscher D and Czarnetzki U 2011 Plasma Sources Sci. Technol. 20 015022

[5] Cornay A, Atomic and Laser Spectroscopy (Oxford: Oxford University Press)

[6] Zatsarinny O and Bartschat K 2006 J. Phys. B: At. Mol. Opt. Phys. 392145

[7] Du B, Celik Y, Luggenhölscher D and Czarnetzki U 2010 Plasma Sources Sci. Technol. 19045008

[8] Pai D Z, Lacoste D A and Laux C O 2010 Plasma Sources Sci. Technol. 19065015

[9] Penache C, Miclea M, Bräuning-Demian A, Hohn O, Schössler S, Jahnke T, Niemax K and SchmidtBöcking H 2002 Plasma Sources Sci. Technol. 11467

[10] Aeschliman D P and Hill R A 1976 Physical Review A 14-4 1421 